\title{
Residencia del tiburón punta blanca de arrecife, Triaenodon obesus, (Carcharhiniformes: Carcharhinidae) en las bahías Chatham y Wafer del Parque Nacional Isla del Coco, Costa Rica
}

\author{
Andrés López-Garro $^{1 *}$, Ilena Zanella ${ }^{1}$, Geiner Golfín-Duarte ${ }^{2} \&$ Maikel Pérez-Montero $^{2}$ \\ 1. Asociación Conservacionista Misión Tiburón, Playas del Coco, Guanacaste, Costa Rica; alopez@misiontiburon.org; \\ izanella@misiontiburon.org \\ 2. Parque Nacional Isla del Coco. Sistema Nacional de Áreas de Conservación, Ministerio del Ambiente, y Energía, \\ Costa Rica; geiner.golfin@sinac.go.cr; elcocolaisla@hotmail.com \\ * Correspondence
}

Recibido 30-I-2019. Corregido 05-V-2019. Aceptado 01-VII-2019.

\begin{abstract}
Residency of the whitetip reef shark (Triaenodon obesus) in Chatham and Wafer Bays, Isla del Coco National Park, Costa Rica. Introduction: The whitetip reef shark (Triaenodon obesus) is a nonmigratory tropical reef species that has shown no high coastal connectivity, as observed for other shark species. At the same time, has the widest distribution of all Indo-Pacific reef shark species. Triaenodon obesus is one of the most common shark species at Isla del Coco National Park (PNIC) and it is present year-round. In the bays located in the north of the PNIC, Chatham Bay and Wafer Bay, there is a large population of whitetip reef sharks. Despite that, information about its residency is scarce. Objective: The aim of this initiative is to study the hábitat use and the movements of the whitetip reef sharks, in order to improve the management of its population at the Isla del Coco National Park and other áreas of Eastern Tropical Pacific. A preliminary study on the residency in the Chatham and Wafer bays of the PNIC was conducted, using acoustic telemetry. Methods: A shark tagging expedition was made to Isla del Coco National Park from November 25 to December 5, 2014. During this expedition, two night trips were made between 6:00 pm and 10:00 pm at Chatham Bay and Wafer Bay. Results: A total of 8 individuals of $T$. obesus were fitted with acoustic tags and two receivers were installed, one in each bay. Sharks were monitored between November 2014 and December 2015, the sharks were detected on 278706 occasions. The sharks remained long periods in the Chatham and Wafer bays. Their average Residency Index (IR) was $0.97 \pm 0.03$, reporting less number of detections during the night hours. Detections of sharks in the bays tend to diminish after 18:00, when the whitetip shark becomes active to hunt on the reef. Conclusions: In conclusion, white tip sharks have a high residence and fidelity, which confirms their low home range. The Protected Marine Area of Isla del Coco probably provides effective protection to the white tip shark population for the illegal fisheries.
\end{abstract}

Key words: Whitetip reef shark, Triaenodon obesus, residence index, acoustic telemetry, Isla del Coco, Eastern Tropical Pacific.

López-Garro, A., Zanella, I., Golfín-Duarte, G., \& Pérez-Montero, M. (2020). Residencia del tiburón punta blanca de arrecife, Triaenodon obesus (Carcharhiniformes: Carcharhinidae) en las bahías Chatham y Wafer del Parque Nacional Isla del Coco, Costa Rica. Revista de Biología Tropical, 68(Supl. 1), S330-S339.

El tiburón punta blanca (Triaenodon obesus) es una especie de arrecife de aguas tropicales, no migratoria, con alta residencia y movimientos limitados (Whitney, Robbins,
Schultzan, Bowen, \& Holland, 2012). A pesar de esto, ha sido reportada en diferentes islas oceánicas a lo largo del Pacífico Este Tropical (Isla del Coco, Malpelo, Galápagos), y posee 
la distribución más amplia de todas las especies de tiburón de arrecife del Indo-Pacífico (Whitney et al., 2012). Distintos autores han reportado como su distribución, ocurrencia y abundancia depende de los corales (Randall, 1977; Ayling \& Choat, 2008).

Triaenodon obesus es una de las especies de tiburón más comunes en el Parque Nacional Isla del Coco (PNIC), Sibaja (2008) observó que no presenta una estacionalidad marcada y que posee una relación negativa con anomalías de la temperatura del agua superficial. Sus hábitos bentónicos o demersales, hacen que $T$. obesus sea observado descansando en el fondo o en cuevas durante el día, mientras que en la noche se convierte en un depredador activo, desplazándose por largos periodos, pasando en movimiento entre el $42 \%$ y el $67 \%$ de su tiempo (Whitney, Papastamatiou, Holland, \& Lowe, 2007).

En las bahías localizadas en el norte del PNIC, Bahía Chatham y Bahía Wafer, reside una saludable población del tiburón punta blanca de arrecife. Estudios previos (conteos subacuáticos) en Bahía Chatham estimaron una abundancia relativa para $T$. obesus de 49.5 \pm 10.4 tiburones/hora (Zanella, López, Golfín, \& Sáenz, 2012). Además, los análisis de los estados reproductivos de los machos que habitan en Bahía Chatham confirmaron que T. obesus no utiliza esta bahía como área de crianza, por el contrario la bahía es un hábitat compartido por adultos, subadultos y juveniles, dominado en su mayoría por adultos (Zanella et al., 2012).

Estudios poblaciones previos de marcaje de captura y recaptura, sugieren que $T$. obesus no realiza grandes movimientos y que prefiere las aguas cristalinas y con cobertura coralinas presentes en Bahía Chatham, con respecto a las aguas turbias de Bahía Wafer (Zanella, López, Martínez, Golfín, \& Morales 2016). A pesar de ser una especie abundante en las bahías Chatham y Wafer del PNIC a lo largo del año, la información sobre su residencia y fidelidad resulta escasa.

Con el fin de mejorar el manejo del tiburón punta blanca de arrecife en aguas del
PNIC y fuera de este, se realizó un estudio preliminar sobre su fidelidad y residencia a las bahías Chatham y Wafer del PNIC, utilizando telemetría acústica.

\section{MATERIALES Y MÉTODOS}

Permiso de Investigación: Todas las actividades de investigación realizadas con el tiburón punta blanca de arrecife fueron amparadas dentro de la RESOLUCION DE INVESTIGACIONES CIENTIFICAS $\mathrm{N}^{\circ}$ ACMIC-PI-004-2013 otorgada por el Área de Conservación Marina Cocos (ACMC) del Sistema Nacional de Áreas de Conservación de Costa Rica (SINAC), Ministerio de Ambiente y Energía (MINAE).

Área de estudio: La Isla del Coco se ubica en aguas oceánicas del Pacífico costarricense $\left(5^{\circ} 32^{\prime} \mathrm{N}-87^{\circ} 04^{\prime} \mathrm{W}\right)$ aproximadamente a 500 $\mathrm{km}$ de la provincia de Puntarenas. En la zona norte de la Isla del Coco, destacan la Bahía Chatham y Bahía Wafer. La Bahía Chatham se ubica entre las coordenadas $5^{\circ} 33^{\prime} 25.28^{\prime \prime} \mathrm{N}$ $87^{\circ} 02^{\prime} 53.11^{\prime \prime}$ W y 5 $33^{\circ} 04.71^{\prime \prime} \mathrm{N}-87^{\circ} 02^{\prime}$ ' $13.76^{\prime}$ " W y tiene una profundidad máxima de $20 \mathrm{~m}$. Se caracteriza por la presencia de aguas cristalinas (con una menor cantidad de nutrientes y sedimentos) y distintas acumulaciones de rocas y arrecifes coralinos. Bahía Wafer se ubica entre las coordenadas $5^{\circ} 32^{\prime} 56.33^{\prime \prime} \mathrm{N}-87^{\circ} 03^{\prime} 44.96^{\prime \prime}$ W y $5^{\circ} 32^{\prime} 47.35^{\prime \prime} \mathrm{N}-87^{\circ} 03^{\prime} 27.48^{\prime \prime}$ W y se caracteriza por ser una bahía con profundidades entre los 10 y $20 \mathrm{~m}$, con la presencia de bancos rocosos y canales arenosos. En la Bahía Wafer desemboca el Río Genio, río de mayor tamaño y con la cuenca de mayor captación de la Isla, por esta razón las aguas de la bahía presentan turbidez y gran cantidad de sedimentos (Sinergia 69, 2002; Sibaja-Cordero, \& Cortés, 2010; Sibaja-Cordero, Troncoso, Benavides, \& Cortés, 2012).

Marcaje de tiburones: Se realizó una expedición de marcaje de tiburones al Parque Nacional Isla del Coco del 25 de noviembre al 5 de diciembre del 2014. Durante esta 
expedición se realizaron 2 giras nocturnas entre las 18:00 y las 22:00 a Bahía Chatham y Bahía Wafer, a bordo de la embarcación del PNIC "Cocos Patrol II".

Los tiburones analizados fueron capturados durante recorridos en las bahías entre las 18:00-21:00 horas. En los sitios de marcaje la profundidad promedio fue de $12.5 \mathrm{~m} \pm 1.5 \mathrm{~m}$. Se utilizó una cuerda de mano con anzuelos circulares \#5 modificados (sin muesca para minimizar el daño físico en el tiburón) con carnada de calamar gigante y calamar torpedo (Loliginidae). Los tiburones capturados fueron subidos a la embarcación por medio de un scoop o Dip Net y se les retiró el anzuelo. Una vez abordo, a cada tiburón se le evaluó el sexo (mediante la presencia o ausencia de los claspers), se le retiró el anzuelo y sucesivamente fue colocado en un ictiómetro, a fin de recolectar sus datos biométricos: la longitud total (LT: medida desde la punta del hocico, hasta el extremo final de la aleta caudal, $\pm 0.1 \mathrm{~cm}$ ) y en el caso de los machos, la longitud de los claspers (LC: medida desde el punto de inserción con la cloaca hasta la punta final de los clasper, $\pm 0.1 \mathrm{~cm})$.

Posterior a la toma de datos biométricos, a cada tiburón capturado se colocó una marca acústica o transmisor. Los transmisores (V13 con $13 \mathrm{~mm}$ de diámetro y $36 \mathrm{~mm}$ de longitud; y V16 con $16 \mathrm{~mm}$ de diámetro y $64 \mathrm{~mm}$ de longitud, Vemco Ltd., Nueva Escocia, Canadá) se implantaron quirúrgicamente en la cavidad peritoneal haciendo una incisión de $2 \mathrm{~cm}$ a lo largo la línea media ventral que luego se cerró a través de suturas quirúrgicas. Los transmisores emiten señales ultrasónicas a $69 \mathrm{kHz}$ y se configuraron para emitir una señal acústica durante un período aleatorio de 40 a 140 segundos; teniendo una duración de batería de aproximada de dos (V13) a cuatro años (V16). Una etiqueta convencional con el número y la información de los investigadores también se colocó externamente en la base de la primera aleta dorsal. Antes de ser devuelto al mar, se le aplicó antibiótico (Neomicina) para disminuir las probabilidades de infecciones. En cada sitio de marcaje, se registró las coordenadas geográficas, la hora de marcaje, la profundidad y temperatura. Los tiburones fueron retenidos por un máximo de tres minutos durante los procedimientos de medición y marcaje.

Anclaje de Receptores Acústicos en el fondo marino: Entre noviembre 2014 y hasta diciembre 2015, dos receptores acústicos VR2 (Vemco Ltd., Halifax, Canadá) fueron anclados en el fondo arenoso en las entradas de las bahías Chatham $\left(05^{\circ} 33\right.$ ' $13.7^{\prime \prime} \mathrm{N}$ y $87^{\circ} 02^{\prime} 32.13$ "W) y Wafer $\left(05^{\circ} 32^{\prime} 42.5 \mathrm{~N}^{\prime \prime}\right.$ y $\left.87^{\circ} 03{ }^{\prime} 46.5^{\prime \prime W}\right)$. Los receptores fueron colocados entre 18-23 m de profundidad utilizando un bloque de concreto $(50-65 \mathrm{~kg}$ ) como anclaje y un cable de nylon (5m) tensado por una boya. Los receptores acústicos se anclaron en el fondo arenoso, con la ayuda de buzos guarda parques expertos. Se determinó el rango de detección de los receptores en $400 \mathrm{~m}$, colocando un transmisor a una distancia variada $(100 \mathrm{~m}, 200 \mathrm{~m}, 300 \mathrm{~m}, 400 \mathrm{~m}$, $500 \mathrm{~m}$ ). El transmisor no fue detectado a una distancia mayor a los $400 \mathrm{~m}$.

Análisis de datos acústicos: La información almacenada en los receptores acústicos colocados en las bahías del PNIC fue descargada utilizando el software VUE versión 2.0.6, aproximadamente cada 3 meses. Los datos acústicos de los tiburones punta blanca marcados en ambas bahías fueron fusionados para analizar la fidelidad de esta especie en las bahías durante diferentes horas del día. Adicionalmente, utilizando la prueba no paramétrica Kruskall Wallis (InFostat versión 1.1), se buscaron diferencias significativas entre el total de detecciones de los tiburones durante las horas del día y la noche en cada bahía.

El índice de residencia fue calculado (IR) para cada tiburón punta blanca a partir del número de días que fue detectado por los receptores (en bahías Chatham y Wafer) y el número total de días que el tiburón estuvo transmitiendo (Papastamatiou, Friedlander, Caselle, \& Lowe, 2010). Este índice tiene como finalidad estandarizar el número de detecciones para cada tiburón, independientemente del periodo de estudio. De tal forma que los tiburones 
marcados que nunca fueron detectados tienen un $\mathrm{IR}=0$, mientras que los tiburones que fueron detectados todos los días desde su marcaje obtuvieron un $\mathrm{IR}=1$. Para todos los tiburones punta blanca marcados se calculó un IR promedio durante todo el periodo de estudio.

\section{RESULTADOS}

Se realizaron dos recorridos nocturnos entre las 18:00 y 21:00 horas para el marcaje de tiburones punta blanca de arrecife. Durante los recorridos nocturnos se capturaron y analizaron tres tiburones T. obesus en Bahía Wafer y cinco T. obesus en Bahía Chatham. La longitud total promedio de los tiburones marcados fue 116.25 $\pm 13.79 \mathrm{~cm}$, con una longitud total máxima de $141.0 \mathrm{~cm}$ y una mínima de $97.0 \mathrm{~cm}$ (Tabla 1).

Entre noviembre 2014 y diciembre 2015 los ocho tiburones punta blanca $T$. obesus marcados fueron detectados por los receptores instalados en las bahías en un total de 278706 ocasiones. De estas, 108205 (30.82\%) en Bahía Wafer y 170501 (61,18\%) detecciones fueron en Bahía Chatham. El promedio de detecciones para cada $T$. obesus marcado fue muy similar en ambas bahías. En Bahía Wafer los $T$. obesus fueron detectados en promedio $36032.67 \pm 19540.42$ ocasiones, mientras en Bahía Chatham el promedio de detecciones para los T. obesus marcados fue $34100.2 \pm$ 19585.69 .

En promedio cada tiburón marcado fue detectado en $34.838,25 \pm 19.607,19$ ocasiones durante el periodo de estudio, sin embargo el número de detecciones para cada tiburón fue variable. Por ejemplo el tiburón "23 523" fue detectado en 20063 ocasiones entre diciembre 2014 y agosto 2015 en Bahía Chatham. Mientras el tiburón con la marca " 23525 " fue detectado en casi 70000 ocasiones durante el mismo periodo en la misma bahía (Tabla 2).

Los ocho tiburones $T$. obesus marcados permanecieron largos periodos en las bahías Chatham y Wafer, así lo revelan las detecciones permanentes en los receptores en el periodo de estudio y lo confirman los Índices de Residencia (IR). En la Bahía Wafer los tiburones marcados fueron detectados en promedio 2770.21 \pm 1940.95 ocasiones cada mes, entre diciembre 2014 y diciembre 2015. Sin embargo algunos tiburones como el "23 527" fueron detectados de manera más permanente en la bahía, siendo detectados en promedio $4489.45 \pm 1563.71$ ocasiones en 12 meses (Fig. 1).

De igual forma en Bahía Chatham los tiburones punta blanca marcados permanecieron por largos periodos siendo detectados en promedio $4171.75 \pm 1031.34$ ocasiones cada mes entre diciembre 2014 y agosto 2015 en

TABLA 1

Datos generales de los tiburones punta blanca de arrecife (Triaenodon obesus) capturados, analizados y marcados en las bahías Chatham y Wafer del PNIC en diciembre 2014

TABLE 1

General data of reef whitetip reef sharks (Triaenodon obesus) caught, analyzed and tagged in the Chatham and Wafer bays of the PNIC in December 2014

\begin{tabular}{cccccccc} 
N & LT $(\mathrm{cm})$ & Sexo & $\mathrm{N}^{\circ}$ Marca Flexi & $\begin{array}{c}\mathrm{N}^{\circ} \text { Marca } \\
\text { Acústica }\end{array}$ & $\begin{array}{c}\text { Hora de } \\
\text { marcaje }\end{array}$ & $\begin{array}{c}\text { Lugar Marcaje } \\
\text { Fecha de Marcaje }\end{array}$ \\
\hline 1 & 129 & H & 291 & 23523 & $20: 10$ & Bahía Chatham & $02 / 12 / 2014$ \\
2 & 97 & H & 284 & 23524 & $18: 50$ & Bahía Chatham & $02 / 12 / 2014$ \\
3 & 112 & H & 292 & 23525 & $20: 25$ & Bahía Chatham & $02 / 12 / 2014$ \\
4 & 122 & M & 290 & 25469 & $18: 32$ & Bahía Chatham & $02 / 12 / 2014$ \\
5 & 141 & H & 281 & 25471 & $19: 45$ & Bahía Chatham & $02 / 12 / 2014$ \\
6 & 108 & M & 492 & 23526 & $19: 30$ & Bahía Wafer & $03 / 12 / 2014$ \\
7 & 111 & M & 469 & 23527 & $20: 20$ & Bahía Wafer & $03 / 12 / 2014$ \\
8 & 110 & M & 496 & 23529 & $20: 35$ & Bahía Wafer & $03 / 12 / 2014$ \\
\hline
\end{tabular}


TABLA 2

Análisis de detecciones de los tiburones punta blanca de arrecife (Triaenodon obesus) registrados en las bahías Chatham y Wafer en el PNIC entre diciembre 2014 y diciembre 2015

TABLE 2

Detections analysis of reef white tip sharks (Triaenodon obesus) recorded in the Chatham and Wafer bays in the PNIC between December 2014 and December 2015

\begin{tabular}{cccccccc} 
N & $\begin{array}{c}\text { Marca } \\
\text { acústica }\end{array}$ & $\begin{array}{c}\text { Fecha de } \\
\text { Marcaje }\end{array}$ & $\begin{array}{c}\text { Total } \\
\text { Detecciones }\end{array}$ & $\begin{array}{c}\text { Última } \\
\text { Detección }\end{array}$ & $\begin{array}{c}\text { Días } \\
\text { Emitiendo }\end{array}$ & $\begin{array}{c}\text { Días } \\
\text { Receptor }\end{array}$ & $\begin{array}{c}\text { IR } \\
1\end{array}$ \\
23523 & $02 / 12 / 2014$ & 20063 & $08 / 08 / 2015$ & 249 & 243 & 0.976 \\
2 & 23524 & $02 / 12 / 2014$ & 35992 & $08 / 08 / 2015$ & 249 & 248 & 0.996 \\
3 & 23525 & $02 / 12 / 2014$ & 69453 & $08 / 08 / 2015$ & 249 & 248 & 0.996 \\
4 & 25469 & $02 / 12 / 2014$ & 12540 & $08 / 08 / 2015$ & 249 & 245 & 0.984 \\
5 & 25471 & $02 / 12 / 2014$ & 32453 & $08 / 08 / 2015$ & 249 & 245 & 0.984 \\
6 & 23526 & $03 / 12 / 2014$ & 38937 & $31 / 12 / 2015$ & 393 & 378 & 0.962 \\
7 & 23527 & $03 / 12 / 2014$ & 58487 & $31 / 12 / 2015$ & 393 & 382 & 0.972 \\
8 & 23529 & $03 / 12 / 2014$ & 10781 & $31 / 12 / 2015$ & 393 & 354 & 0.900 \\
\hline
\end{tabular}

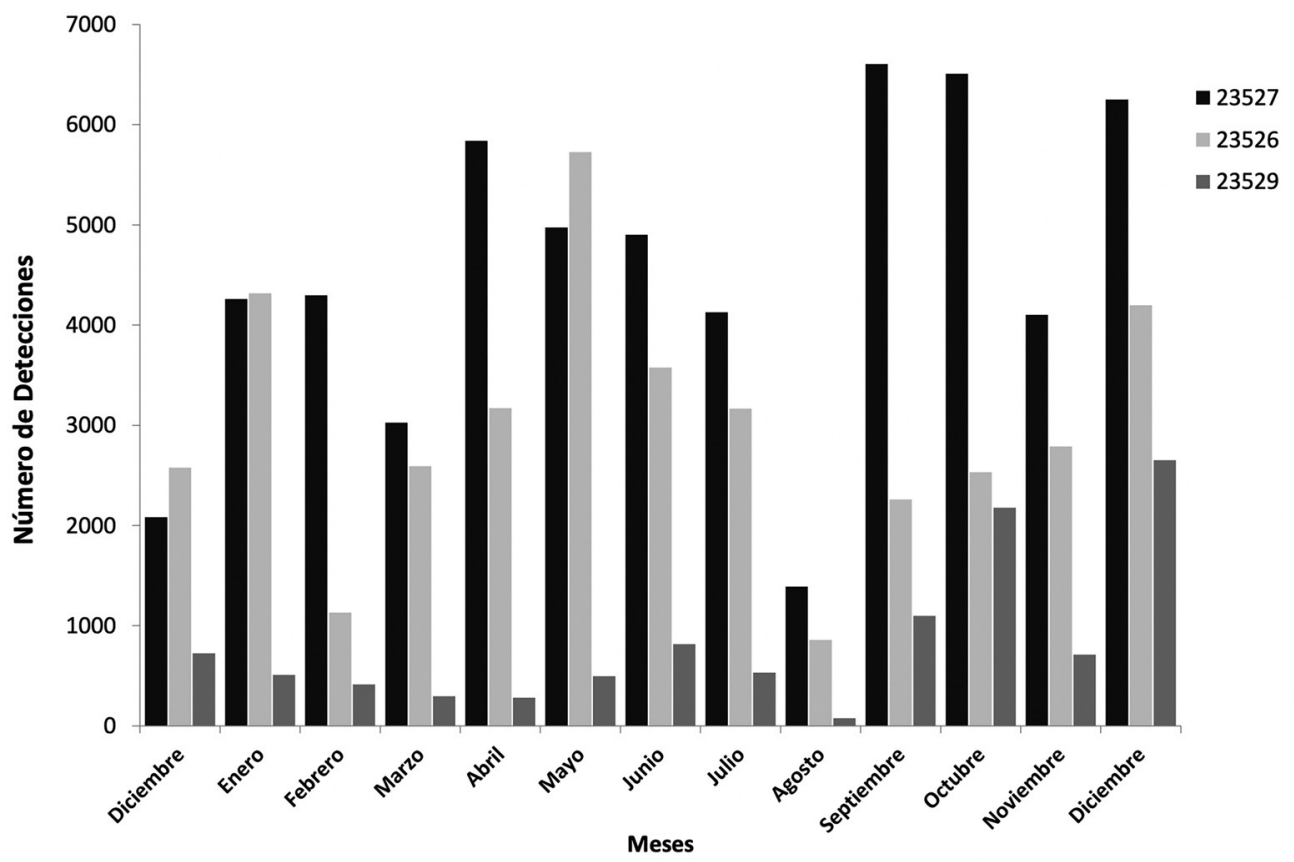

Fig. 1. Número de detecciones registradas por mes por los tiburones punta blanca de arrecife (Triaenodon obesus) en Bahía Wafer, Parque Nacional Isla del Coco, Costa Rica.

Fig. 1. Number of detections recorded per month by white tip reef sharks (Triaenodon obesus) in Wafer Bay, Isla del Coco National Park, Costa Rica. 


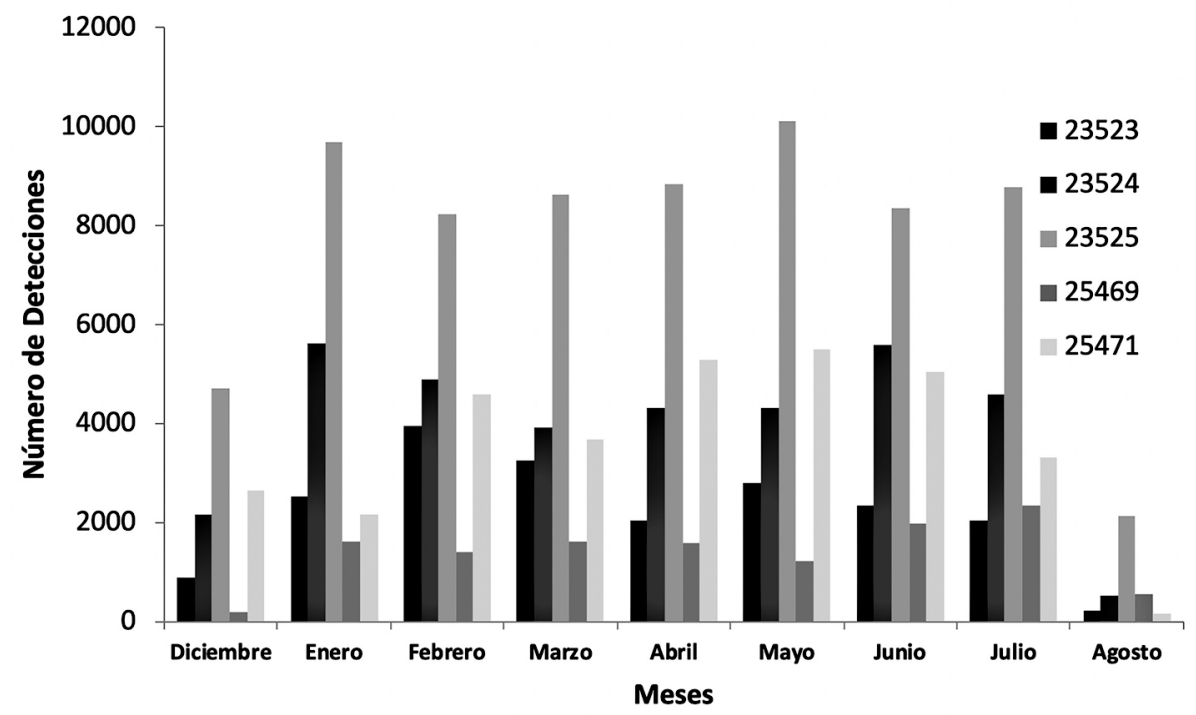

Fig 2. Número de detecciones registradas por mes por los tiburones punta blanca de arrecife (Triaenodon obesus) en Bahía Chatham, Parque Nacional Isla del Coco, Costa Rica.

Fig. 2. Number of detections recorded per month by white tip reef sharks (Triaenodon obesus) in Chatham Bay, Isla del Coco National Park, Costa Rica.

la bahía. Sobresale el tiburón " 23525 ” quien fue detectado en más de 8000 ocasiones por mes en Bahía Chatham, durante nueve meses (Diciembre 2014-Agosto 2015) (promedio de detecciones $=8416.50 \pm 1520.95 / \mathrm{mínimo}=$ 4714 detecciones, máximo=10096) (Fig. 2).

El IR promedio de los T. obesus marcados fue de $0.97 \pm 0.03$. Los tiburones $T$. obesus "23 524" y "23 525" fueron registrados todos los días durante más de nueve meses en la Bahía Chatham, siendo registrados en la bahía por más de 70 días consecutivos. Sobresale el tiburón T. obesus "23526" que obtuvo un IR (0.962) y fue detectado en Bahía Wafer continuamente durante 240 días. (Tabla 2).

En cuanto a la permanencia en las bahías Chatham y Wafer de los ocho tiburones T. obesus marcados durante las horas del día, hubo una mayor cantidad de detecciones en horas diurnas. En las Fig. 3 y Fig. 4 se observa una tendencia en la baja del número total de detecciones a partir de las 18:00 horas, sin embargo, las diferencias entre las detecciones de las horas del día y de la noche solo resulta significativa solo para Bahía Wafer $(\mathrm{H}=17.16 ; \mathrm{p}<0.0001)$,

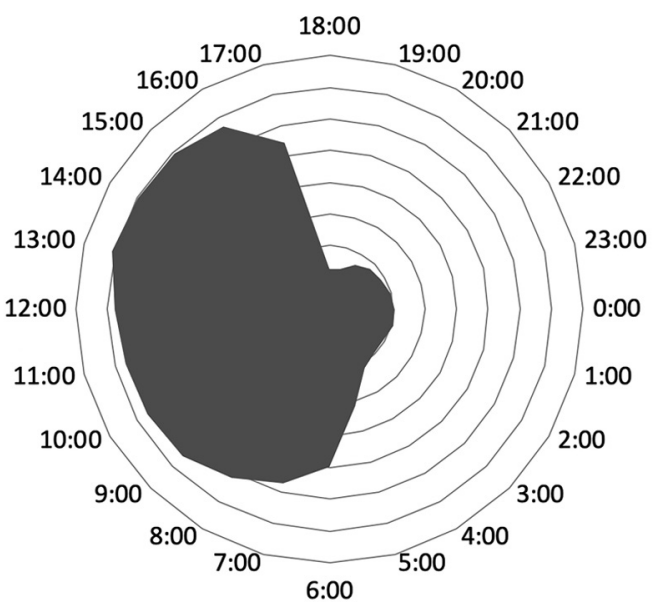

Fig. 3. Número de detecciones registradas de tiburones punta blanca de arrecife (Traienodon obesus) expresadas en porcentajes del total detecciones $(\mathrm{N}=108205$ detecciones $)$ en Bahía Wafer, Parque Nacional Isla del Coco, Costa Rica. Cada anillo representa 30000 detecciones, el gráfico tiene 8 anillos en total.

Fig. 3. Number of detections recorded by white tip reef sharks (Triaenodon obesus) expressed as percentage of the total number of detections $(\mathrm{N}=108205)$, in Wafer Bay, Isla del Coco National Park, Costa Rica. In this graph each ring represents 30000 detections, the graph has 8 rings in total. 


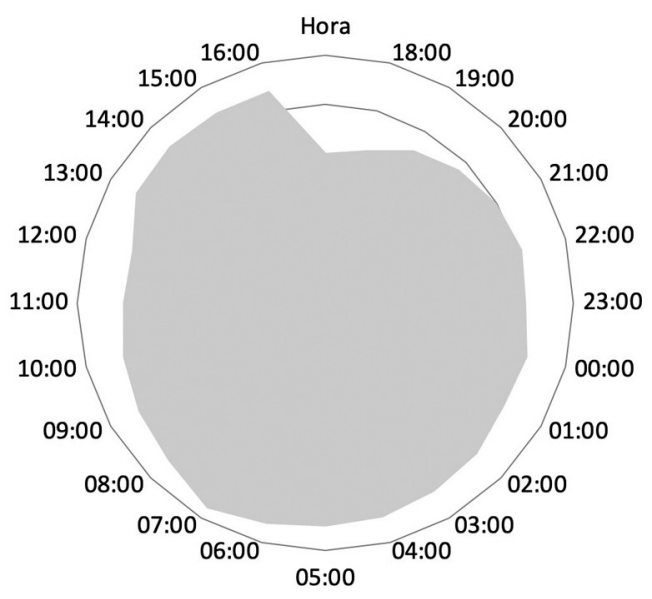

Fig. 4. Número de detecciones registradas expresadas en porcentajes del total detecciones ( $\mathrm{N}=170501$ detecciones) en Bahía Chatham, Parque Nacional Isla del Coco, Costa Rica. En este gráfico cada anillo representa el 50000 detecciones, la figura tiene 5 anillos en total.

Fig. 4. Number of detections recorded by white tip reef sharks (Triaenodon obesus) expressed as percentage of the total number of detections $(\mathrm{N}=170501)$ in Chatham Bay, Isla del Coco National Park, Costa Rica. Each ring represents 50000 detections, there are 5 rings in total.

no encontrándose diferencias significativas para Bahía Chatham $(\mathrm{H}=3.00 ; \mathrm{p}=0.083)$.

Durante el periodo de estudio solamente uno de los tiburones marcados ("25427") ha cruzado entre las bahías y lo hizo solamente en una ocasión desde BahíaWafer hasta Bahía Chatham. La gran variación en el número de detecciones entre tiburones, nos muestra como ciertos individuos permanecen en las bahías todo el día, mientras otros, realizan movimientos cortos posiblemente con fines alimenticios.

\section{DISCUSIÓN}

El alto número de detecciones y la permanencia de todos tiburones $T$. obesus marcados en ambas bahías durante el periodo de estudio, permitió conocer sobre la residencia de esta especie tan abundante en el PN Isla del Coco. T. obesus es una especie de arrecife que depreda activamente entre rocas y corales (Randal, 1977), por lo que la implantación interna de la marcas acústicas disminuyó la posibilidad de desprendimiento de los transmisores. Peñaherrera, Hearn \& Kuhn (2012) marcaron con telemetría acústica $T$. obesus en las Islas Galápagos, colocando los transmisores acústicos externamente utilizando una arbaleta modificada durante inmersiones a pulmón. De los nueve tiburones que marcaron, cuatro perdieron los transmisores en poco tiempo. En el presente estudio todos los tiburones marcados transmitieron exitosamente en las bahías de Chatham y Wafer del Parque Nacional Isla del Coco durante más de doce meses.

Los tiburones punta blanca de arrecife mostraron una alta fidelidad al sitio de marcaje, que se ve reflejado en los elevados índices de residencia reportados (Tabla 1). El índice de residencia promedio $(0.97 \pm 0.03)$ estimado en el presente estudio para $T$. obesus fue particularmente alto en comparación con otras especies de tiburón de arrecife. Por ejemplo, Espinoza, Heupel, Tobin, \& Simpfendorfer (2015) colocaron transmisores acústicos en 40 individuos del tiburón gris de arrecife, Carcharhinus amblyrhynchos, en la Gran Barrera de Arrecife Australiana y estimaron un índice de residencia promedio de $0.78 \pm 0.26$. Esta disminución en el IR, probablemente se debe a que especies de tiburones de arrecife de mayor tamaño como $C$. amblyrhynchos poseen necesidades fisiológicas y gastos energéticos mayores, por lo cual tienden a ampliar su rango de hogar y residencia con la ontogenia, utilizando áreas específicas como criaderos (Garla, Chapman, Wetherbee, \& Shivji, 2006). Esto probablemente no ocurre u ocurre en menor grado, con el tiburón punta blanca de arrecife. De hecho, Zanella et al. (2012) reportaron distintos estados de madurez en individuos capturados en un estudio de captura y re-captura en bahía Chatham, concluyendo que T.obesus no utiliza la bahía como área de crianza, por el contrario es un hábitat compartido por adultos, subadultos y juveniles, dominado en su mayoría por adultos.

El alto y continuo número de detecciones registrado por los receptores en las bahías durante el periodo de estudio de todos los tiburones marcados, nos confirma que $T$. obesus es una especie que permanecen por largos 
periodos en las aguas protegidas y someras de las bahías. Incluso algunos tiburones como "23526" fueron detectados durante 240 días consecutivos en Bahía Chatham y solamente uno de los tiburones marcados, el tiburón “23 527”'registró un movimiento entre ambas bahías. A diferencia, Peñaherrera et al. (2012) en las Islas Galápagos reportaron para T. obesus periodos de residencia menores (máximo tres meses), y solo dos tiburones demostraron altos niveles de fidelidad, sugiriendo que el tiburón punta blanca de arrecife en las Islas Galápagos puede tener varios sitios preferidos dentro de su rango de hogar.

Las detecciones de los tiburones en las bahías tienden a disminuir a partir de las 18:00 horas, cuando el tiburón punta blanca se vuelve activo para cazar en el arrecife. Esta tendencia es significativa solo para Bahía Wafer, donde los arrecifes son menos estructurados y probablemente ofrezcan menos alimento a los tiburones. Como consecuencia, es probable que los $T$. obesus marcados en Wafer amplíen su rango de hogar hacia otros sitios de la isla, adyacentes a la bahía. Otros autores han reportado la misma tendencia, por ejemplo, el tiburón de arrecife del Caribe (Carcharhinus perezi) en el Archipiélagos de Fernando de Noronha, en Brazil, amplía sus movimientos durante las horas nocturnas (Garla et al., 2006) en busca de alimento.

En conclusión, los tiburones punta blanca poseen una alta residencia y fidelidad, lo cual confirma su bajo rango de hogar. El Área Marina Protegida de la Isla del Coco probablemente brinda una protección efectiva a la población del tiburón punta blanca. De hecho, la pesca ilegal en este Parque Nacional se concentra entre las 8 y 12 millas de la isla, motivo por lo cual esta especie se reporta aisladamente faenas ilegales (López, Zanella, Martínez, Golfín \& Pérez 2016). Es importante tener claro que aunque la pesca ilegal no es su mayor amenaza, la abundancia de T. obesus ha declinado en los últimos 30 años, según lo reportan sus avistamientos en el buceo en el PN Isla del Coco (White et al. 2015). Lo mismo está sucediendo en la Gran Barrera Coralino donde se reporta una reducción del $7 \%$ anual de los avistamientos de Triaenodon obesus (Mukharror et al., 2017).

A pesar de no ser impactada por la pesca, sí es muy vulnerable a los cambios ambientales y de hábitat. Espinoza, Cappo, Heupel, Tobin, \& Simpfendorfer (2014) demostraron como la cobertura coralina afecta la abundancia de las especies de tiburón de arrecife, como T. obesus. Como consecuencia de su baja movilidad y su alto aislamiento geográfico, las anomalías de temperatura cada vez más recurrentes en nuestra región y la pérdida de cobertura coralina, podrían generar en un deterioro genético poblacional en el tiburón punta blanca de arrecife presente en el AMP del PNIC. Por lo tanto, se recomienda ampliar este estudio y mejorar el monitoreo de esta población, así como realizar análisis genéticos para determinar su salud.

Declaración de ética: los autores declaran que todos están de acuerdo con esta publicación y que han hecho aportes que justifican su autoría; que no hay conflicto de interés de ningún tipo; y que han cumplido con todos los requisitos y procedimientos éticos y legales pertinentes. Todas las fuentes de financiamiento se detallan plena y claramente en la sección de agradecimientos. El respectivo documento legal firmado se encuentra en los archivos de la revista.

\section{AGRADECIMIENTOS}

Agradecemos al Área de Conservación Marina Isla del Coco (ACMC) y a los funcionarios del Parque Nacional Isla del Coco por habernos apoyado durante las expediciones con recursos, personal y embarcaciones. Solo su compromiso y dedicación permitió concluir con el marcaje tan exitosamente. Muchas gracias a Keylor, Isaac, Geiner, Maikel, Lucas, Geovanny, Victor, Esteban, Sam, Manuel, A la vez, la investigación fue posible gracias a los fondos aportados por Fundación Amigos de la Isla del Coco (FAICO). 


\section{RESUMEN}

Introducción: El tiburón punta blanca (Triaenodon obesus) es una especie de arrecife de aguas tropicales, no migratoria, que ha demostrado no tener una alta conectividad costera, como la observada para otras especies de tiburón, sin embargo posee la distribución más amplia de todas las demás especies de tiburón de arrecife del Indo-Pacífico. T. obesus, es una de las especies de tiburón más comunes en el Parque Nacional Isla del Coco (PNIC), y no presenta una estacionalidad marcada. En las bahías localizadas en el norte del PNIC, Bahía Chatham y Bahía Wafer, reside una saludable población del tiburón punta blanca de arrecife. A pesar de ser una especie abundante en las bahías Chatham y Wafer del PNIC, la información sobre su residencia resulta escaza. Objetivo: Conocer sobre el uso de hábitat y los movimientos del tiburón punta blanca de arrecife mejorará su manejo en el PNIC y en otros lugares del Pacífico Tropical Oriental. Para esto se realizó un estudio preliminar sobre la residencia en las bahías Chatham y Wafer del PNIC, utilizando telemetría acústica. Métodos: Se realizó una expedición de marcaje de tiburones al Parque Nacional Isla del Coco entre el 25 de noviembre al 5 de diciembre del 2014. Durante esta expedición se realizaron 2 giras nocturnas entre las 18:00 y las 22:00 a Bahía Chatham y Bahía Wafer y se marcaron 8 individuos de T. obesus con telemetría acústica y se instalaron dos receptores, uno en cada bahía. Resultados: Entre noviembre 2014 y diciembre 2015 los tiburones punta blanca marcados fueron detectados por los receptores instalados en las bahías en un total de 278706 ocasiones. Los tiburones T. obesus marcados permanecieron largos periodos en las bahías Chatham y Wafer, El índice de Residencia (IR) medio de los T. obesus marcados fue de $0.97 \pm 0,03$, reportando una tendencia en la baja del número total de detecciones durante las horas nocturnas. Los tiburones punta blanca de arrecife mostraron una alta fidelidad al sitio de marcaje, que se ve reflejado en los elevados índices de residencia reportados, particularmente altos en comparación con otras especies de tiburón de arrecife. Las detecciones de los tiburones en las bahías tienden a disminuir a partir de las 18:00 horas, cuando el tiburón punta blanca se vuelve activo para cazar en el arrecife. Conclusiones: En conclusión, los tiburones punta blanca poseen una alta residencia y fidelidad, lo cual confirma su bajo rango de hogar. El Área Marina Protegida de la Isla del Coco probablemente brinda una protección efectiva a la población del tiburón punta blanca de la pesca ilegal.

Palabras claves: Tiburón punta blanca de arrecife, Triaenodon obesus, índice de residencia, telemetría acústica, Isla del Coco, Pacífico Tropical Oriental.

\section{REFERENCIAS}

Ayling, A., \& Choat, J. (2008). Abundance patterns of reef sharks and predatory fishes on differently zoned reefs in the offshore Townsville region: Final Report to the Great Barrier Reef Marine Park Authority, Queensland, Australia.

Espinoza, M., Heupel, M. R., Tobin, A. J., \& Simpfendorfer, C. A. (2015). Residency patterns and movements of grey reef sharks (Carcharhinus amblyrhynchos) in semi-isolated coral reef habitats. Marine biology, 162(2), 343-358.

Espinoza, M., Cappo, M., Heupel, M., Tobin, A. J. \& Simpfendorfer, C. A. (2014). Quantifying shark distribution patterns and species-habitat associations: implications of Marine Park Zoning. PLoS ONE, 9, e106885. doi: 10.1371/journal.pone.0106885

Garla, R. C., Chapman, D. D., Wetherbee, B. M., \& Shivji, M. (2006). Movement patterns of young Caribbean reef sharks, Carcharhinus perezi, at Fernando de Noronha Archipelago, Brazil: the potential of marine protected areas for conservation of a nursery ground. Marine Biology, 149, 189-199.

López-Garro, A., Zanella, I., Martínez, F., Golfín-Duarte, G. \& Pérez-Montero, M. (2016). La pesca ilegal en el Parque Nacional Isla del Coco, Costa Rica. Revista de Biología Tropical, 64(Suplemento 1), 249-261.

Mukharror, D. A., Tiara B. I., Ichsan, T. B., Muhammad, P. N., \& Triutami, S. (2017). Correlation Between Existence of Reef Sharks with Abundance of Reef Fishes in South Waters of Morotai Island (North Moluccas). IOP Conf. Series: Earth and Environmental Science, 89, 012006.

Papastamatiou, Y. P., Friedlander, A. M., Caselle, J. E., \& Lowe, C. G. (2010). Long-term movement patterns and trophic ecology of blacktip reef sharks (Carcharhinus melanopterus) at Palmyra Atoll. Journal of Experimental Marine Biology and Ecology, 386(1), 94-102.

Peñaherrera, C., Hearn, A. R., \& Kuhn, A. (2012). Diel use of a saltwater creek by white-tip reef sharks Triaenodon obesus (Carcharhiniformes: Carcharhinidae) in Academy Bay, Galapagos Islands. Revista de Biología Tropical, 60(2), 735-743.

Randall, J. E. (1977). Contribution to the biology of the white-tip reef sharks (Triaenodon obesus). Pacific Science, 31, 143-164.

Robbins, W. D. (2006). Abundance, demography and population structure of the grey reef shark (Carcharhinus amblyrhinchos) and the white-tip reef shark (Triaenodon obesus) (Fam. Carcharhinidae). Ph.D. Thesis, James Cook University, Townsville, Australia.

Sibaja, J. (2008). Tendencias espacio-temporales de los avistamientos de fauna marina en los buceos turísticos (Isla del Coco, Costa Rica). Revista de Biología Tropical, 56(Suplemento 2), 113-132. 
Sibaja-Cordero, J. A., \& Cortés, J. (2010). Comparación temporal de la composición y zonación de organismos en el intermareal rocoso del Parque Nacional Isla del Coco, Pacífico de Costa Rica. Revista de Biología Tropical, 58(Suplemento 4), 1387-1403.

Sibaja-Cordero, J. A., Troncoso, J., Benavides-Varela, C., \& Cortés, J. (2012). Distribution of shallow water soft and hard bottom seabeds in the Isla del Coco National Park, Pacific Costa Rica. Revista de Biología Tropical, 60(Suplemento 3), 53-66.

Sinergia 69 (2002). Anexo V: Caracterización de las bahías de Chatham y Wafer e inventario preliminar de especies marinas. Area de Conservación Marina Isla del Coco (ACMIC). Proyecto: Estudio de factibilidad y diseño para la construcción de infraestructura y servicios de apoyo en el Parque Nacional Isla del Coco. San José, Costa Rica: Sinergia.

Speed, C. W., Meekan, M. G., Field, I. C., McMahon, C. R., Stevens, J. D., McGregor, F., Huveneers, C., Berger, Y., \& Bradshaw, C. J.A. (2011). Spatial and temporal movement patterns of a multi-species coastal reef shark aggregation. Marine Ecology Progress Series, 429, 261-275.
Whitney, N., Papastamatiou, Y., Holland, K., \& Lowe, C. (2007). Use of an acceleration data logger to measure diel activity patterns in captive whitetip reef sharks, Triaenodon obesus. Aquatic Living Resources ,20, 299-305.

Whitney, N. M., Robbins, W. D., Schultz, J. K., Bowen, B. W., \& Holland K. (2012). Oceanic dispersal in a sedentary reef shark (Triaenodon obesus): genetic evidence for extensive connectivity without a pelagic larval stage. Journal of Biogeography, 39, 1144-115.

Zanella, I., López, A., Golfín, G., \& Sáenz, J. (2012). Abundancia, tamaño y estructura poblacional del tiburón punta blanca de arrecife, Triaenodon obesus (Carcharhiniformes: Carcharhinidae), en Bahía Chatham, Parque Nacional Isla del Coco, Costa Rica. Revista de Biología Tropical, 60(Suplemento 3), 339-346.

Zanella, I., López-Garro, A., Martínez, F., Golfín-Duarte, G., \& Morales, K. (2016). Poblaciones de tiburones en las bahías Chatham y Wafer del Parque Nacional Isla del Coco, Costa Rica. Revista de Biología Tropical, 64(Suplemento 1), 177-186. 